\title{
Suppementary Material
}

\section{VALRAY User's Manual}

\author{
R. F. Stewart M. A. Spackman C. Flensburg \\ Carnegie Mellon University \& University of Copenhagen
}

Version: 2.1 July 28, 2000 


\section{Electrostatic Properties from Pseudoatom Density Functions}

The gsf's in VALRAY are Fourier transforms of analytical functions which consist of radial functions, given in Appendix A, and spherical surface harmonics. For pseudoatom $p$, the electron density part consists of the bases,

$$
\rho_{p, \ell m \pm}(\mathbf{r})=\rho_{p, \ell}(r) y_{\ell m \pm}\left(\Omega_{\mathbf{r}}\right) / 4 \pi
$$

with a corresponding basis for gsf $p$,

$$
f_{p, \ell m \pm}(\mathbf{K})=i^{\ell} f_{p, \ell}(K) y_{\ell m \pm}\left(\Omega_{\mathbf{K}}\right)
$$

With the use of population coefficients, $C_{p \ell m \pm}$, the full pseudoatom density is,

$$
\rho_{p}(\mathbf{r})=\sum_{\ell m \pm} C_{p \ell m \pm} \rho_{p, \ell m \pm}(\mathbf{r})
$$

or the full gsf at site $p$ is,

$$
f_{p}(\mathbf{K})=\sum_{\ell m \pm} C_{p \ell m \pm} f_{p, \ell m \pm}(\mathbf{K})
$$

The pseudoatoms in superposition at sites $\mathbf{R}_{p}$ may be employed to represent the density of a molecule or crystal,

$$
\rho(\mathbf{r})=\sum_{p} \rho_{p}\left(\mathbf{r}-\mathbf{R}_{p}\right)
$$

or equivalently, the gsf's can be used to construct a form factor or crystal structure factor,

$$
\mathrm{F}(\mathbf{K})=\sum_{p} f_{p}(\mathbf{K}) e^{i \mathbf{K} \cdot \mathbf{R}_{p}}
$$

Either (92) or (93) may be used for the derivation of electrostatic properties of molecules and crystals. The present appendix will outline the conversion of (88) or (89) into components for the electrostatic potential, electrical field, electrical field gradients, gradient of the electron density, the hessian of the electron density, and the Laplacian of the electron density. Although some useful relations for mean thermal electrical properties have been developed, the results presented here are restricted to electrostatic properties based on stationary pseudoatoms.

An irregular spherical harmonic, which is

$$
V_{L M \pm}=r^{-(L+1)} y_{L M \pm}\left(\Omega_{\mathbf{r}}\right)
$$

Ed. 2.1 July 28, 2000 
has the pseudoatom expectation value of,

$$
\begin{aligned}
\left\langle V_{L M \pm}\right\rangle & =\int \rho_{p}\left(\mathbf{r}+\mathbf{R}_{p}\right) r^{-(L+1)} y_{L M \pm}\left(\Omega_{\mathbf{r}}\right) d^{3} \mathbf{r} \\
& =\left(\frac{i^{-L}}{2 \pi^{2}(2 L-1) ! !}\right) \int f_{p}(\mathbf{K}) e^{-i \mathbf{K} \cdot \mathbf{R}_{p}} y_{L M \pm}\left(\Omega_{\mathbf{K}}\right) K^{L-2} d^{3} \mathbf{K}
\end{aligned}
$$

The charge density is centered at $-\mathbf{R}_{p}$. For $\left|\mathbf{R}_{p}\right|$ equal to zero, $\left\langle V_{L M \pm}\right\rangle$ will vanish unless $f_{p}(\mathbf{K})$ has a spherical surface harmonic $y_{\ell m \pm}$ with $\ell=L$ and $m \pm=M \pm$. With $\left|\mathbf{R}_{p}\right|$ not equal to zero, the products $y_{\ell m \pm}\left(\Omega_{\mathbf{K}}\right) y_{L M \pm}\left(\Omega_{\mathbf{K}}\right)$ will be a linear combination of other spherical surface harmonics that will match the harmonics of the plane wave in integration over $\Omega_{\mathbf{K}}$. We will use (96) to derive the pseudoatom contribution to the potential, electrical field, and electric field gradient at the site $\mathbf{R}_{p}$ from the pseudoatom center.

\section{C.1 Electrostatic Potential}

For $L=0$ (96) will give the electrostatic potential at $\mathbf{R}_{p}$. The $y_{00}$ in (96) is unity for all $\mathbf{K}$ so the angular integration gives $4 \pi \times y_{\ell m \pm}\left(\Omega_{\mathbf{R}_{p}}\right)$. With $L=0$ (96) is reduced to evaluation of the radial integral in $K$,

$$
\frac{2}{\pi} \int_{0}^{\infty} f_{p, \ell}(K) j_{\ell}\left(K R_{p}\right) d K=\int_{0}^{\infty} \rho_{p, \ell}(x) x^{2}\left(\frac{2}{\pi} \int_{0}^{\infty} j_{\ell}(K x) j_{\ell}\left(K R_{p}\right) d K\right) d x
$$

The integral in the parentheses of eqn (97) is known as the discontinuous integral of Weber-Schafheitlin. The eplicit solutions in terms of $x$ and $\mathbf{R}_{p}$ is found in Watson [50] eqns (2) and (3) on p.401. Thus the integral with the two spherical Bessel functions in the integrand of (97) is,

$$
\frac{2}{\pi} \int_{0}^{\infty} j_{\ell}(K x) j_{\ell}\left(K R_{p}\right) d K=\frac{1}{2 \ell+1}\left\{\begin{array}{cl}
\frac{x^{\ell}}{R_{p}^{\ell+1}} & 0<x<R_{p} \\
\frac{R_{p}^{\ell}}{x^{\ell+1}} & R_{p}<x<\infty
\end{array}\right.
$$

From (98) the double integral of (97) reduces to a radial function in $R_{p}$,

$$
\mathcal{R}_{p, \ell}\left(R_{p}\right)=\frac{1}{2 \ell+1}\left(\frac{1}{R_{p}^{\ell+1}} \int_{0}^{R_{p}} \rho_{p, \ell}(x) x^{\ell+2} d x+R_{p}^{\ell} \int_{R_{p}}^{\infty} \rho_{p, \ell}(x) x^{1-\ell} d x\right)
$$

The electrostatic potential due to a pseudoatom centered at $-\mathbf{R}_{p}$ is,

$$
\phi_{p}\left(\mathbf{R}_{p}\right)=\sum_{\ell m \pm} C_{p \ell m \pm} \mathcal{R}_{p, \ell}\left(R_{p}\right) y_{\ell m \pm}\left(\Omega_{\mathbf{R}_{p}}\right)
$$

It is (100) which is coded into one of the FOPROP programs in the VALRAY package.

Ed. 2.1 July 28,2000 


\section{C.2 Electrical Field}

The $L=1$ case for $\left\langle V_{L M \pm}\right\rangle$, given by (96), is a vector quantity. It is the electric field at $\mathbf{R}_{p}$ generated by a pseudoatom. The $y_{L M \pm}\left(\Omega_{\mathbf{K}}\right)$ in (96) have $L=1$ and $M \pm=1+, 1-, 0$. The products of these functions with the gsf surface harmonics, $y_{\ell m \pm}$, generate linear combinations of other harmonics. A gsf with an harmonic that is degree $\ell$ will generate harmonics of degree $\ell+1$ and $\ell-1$ for the angular variable $\Omega_{\mathbf{R}_{p}}$. Some of these may be zero, since the associated Legendre vanishes if $\ell \pm 1$ is less than $|m \pm 1|$. For those surface harmonics that are non-zero, the angular integration over $\Omega_{\mathbf{K}}$ will generate $\pm 4 \pi \times y_{n k \pm}\left(\Omega_{\mathbf{R}_{p}}\right)$ surface harmonics with $n=\ell \pm 1$ and $k=m \pm 1$. Since $f_{p, \ell}(K)$ is the Fourier-Bessel transform of $\rho_{p, \ell}$, the radial integrals over $K$ will be:

$$
\begin{aligned}
\frac{2}{\pi} \int_{0}^{\infty} f_{p, \ell}(K) j_{\ell+1} & \left(K R_{p}\right) K d K \\
= & \int_{0}^{\infty} \rho_{p, \ell}(x) x^{2}\left(\frac{2}{\pi} \int_{0}^{\infty} j_{\ell}(K x) j_{\ell+1}\left(K R_{p}\right) K d K\right) d x \\
\frac{2}{\pi} \int_{0}^{\infty} f_{p, \ell}(K) j_{\ell-1}( & \left.K R_{p}\right) K d K \\
= & \int_{0}^{\infty} \rho_{p, \ell}(x) x^{2}\left(\frac{2}{\pi} \int_{0}^{\infty} j_{\ell}(K x) j_{\ell-1}\left(K R_{p}\right) K d K\right) d x
\end{aligned}
$$

Both (101) and (102) contain the discontinuous integrals of Weber-Schafheitlin with explicit solutions:

$$
\begin{aligned}
& \frac{2}{\pi} \int_{0}^{\infty} j_{\ell}(K x) j_{\ell+1}\left(K R_{p}\right) K d K= \begin{cases}\frac{x^{\ell}}{R_{p}^{\ell+2}} & 0<x<R_{p} \\
0 & R_{p}<x<\infty\end{cases} \\
& \frac{2}{\pi} \int_{0}^{\infty} j_{\ell}(K x) j_{\ell-1}\left(K R_{p}\right) K d K= \begin{cases}0 & 0<x<R_{p} \\
\frac{R_{p}^{\ell-1}}{x^{\ell+1}} & R_{p}<x<\infty\end{cases}
\end{aligned}
$$

(101) and (103) combine to make the radial function,

$$
\mathcal{R}_{p, \ell+1}\left(R_{p}\right)=\frac{1}{R_{p}^{\ell+2}} \int_{0}^{R_{p}} \rho_{p, \ell}(x) x^{\ell+2} d x
$$

as do (102) and (104) form the companion,

$$
\mathcal{R}_{p, \ell-1}\left(R_{p}\right)=R_{p}^{\ell-1} \int_{R_{p}}^{\infty} \rho_{p, \ell}(x) x^{1-\ell} d x
$$


The $\ell+1$ type radial function (105) is combined with the $\ell+1$ surface harmonic as is the $\ell-1$ (106) with the $\ell-1$ harmonic to give the electric field at $\mathbf{R}_{p}$ due to a gsf $f_{p}(\mathbf{K})$ :

$$
\begin{aligned}
& E_{X_{p}}= \\
& \sum_{\ell m \pm} \frac{-C_{p \ell m \pm}}{2(2 \ell+1)}\left[\mathcal{R}_{p, \ell+1}\left(R_{p}\right)\left(y_{\ell+1 m+1 \pm}\left(\Omega_{\mathbf{R}_{p}}\right)-\frac{(\ell-m+2) !}{(\ell-m) !} y_{\ell+1 m-1 \pm}\left(\Omega_{\mathbf{R}_{p}}\right)\right)\right. \\
& \left.+\mathcal{R}_{p, \ell-1}\left(R_{p}\right)\left(y_{\ell-1 m+1 \pm}\left(\Omega_{\mathbf{R}_{p}}\right)-\frac{(\ell+m) !}{(\ell+m-2) !} y_{\ell-1 m-1 \pm}\left(\Omega_{\mathbf{R}_{p}}\right)\right)\right] \quad(107) \\
& E_{Y_{p}=} \\
& \sum_{\ell m \pm} \frac{\mp C_{p \ell m \pm}}{2(2 \ell+1)}\left[\mathcal{R}_{p, \ell+1}\left(R_{p}\right)\left(y_{\ell+1 m+1 \mp}\left(\Omega_{\mathbf{R}_{p}}\right)+\frac{(\ell-m+2) !}{(\ell-m) !} y_{\ell+1 m-1 \mp}\left(\Omega_{\mathbf{R}_{p}}\right)\right)\right. \\
& \left.+\mathcal{R}_{p, \ell-1}\left(R_{p}\right)\left(y_{\ell-1 m+1 \mp}\left(\Omega_{\mathbf{R}_{p}}\right)+\frac{(\ell+m) !}{(\ell+m-2) !} y_{\ell-1 m-1 \mp}\left(\Omega_{\mathbf{R}_{p}}\right)\right)\right] \quad(108) \\
& E_{Z_{p}}=\sum_{\ell m \pm} \frac{-C_{p \ell m \pm}}{(2 \ell+1)}\left[\mathcal{R}_{p, \ell+1}\left(R_{p}\right)(\ell-m+1) y_{\ell+1 m \pm}\left(\Omega_{\mathbf{R}_{p}}\right)\right. \\
& \left.-\mathcal{R}_{p, \ell-1}\left(R_{p}\right)(\ell+m) y_{\ell-1 m \pm}\left(\Omega_{\mathbf{R}_{p}}\right)\right]
\end{aligned}
$$

The expressions for the $X_{p}, Y_{p}$ and $Z_{p}$ components of the electric field, given above by (107), (108) and (109), respectively, are encoded into FOPROP for the calculation of the electric field at $\mathbf{R}_{p}$ generated by pseudoatom $p$.

\section{C.3 Electrical Field Gradients}

The electric field gradient of a charge density distribution is related to the second derivatives of its potential, $\phi(\mathbf{r})$. It is a traceless, second rank tensor.

$$
\mathrm{Q}_{\alpha \beta}=\frac{\partial^{2} \phi(\mathbf{r})}{\partial \alpha \partial \beta}-\frac{\delta_{\alpha \beta}}{3} \nabla^{2} \phi(\mathbf{r})
$$

It is easy to recast (110) in terms of $K$-space. In this case the electric field gradient, due to pseudoatom $p$, may be determined from (96) with $L=2$. A gsf will form linear combinations of harmonics with degree $\ell+2, \ell$, and $\ell-2$ via multiplication by the operator $y_{2 M \pm}\left(\Omega_{\mathbf{K}}\right)$. Angular integration will generate $4 \pi \times i^{-j}$, with $j=4,2$, and 0 for the $\ell+2, \ell$, and $\ell-2$ harmonics, respectively.

Ed. 2.1 July 28,2000 
The corresponding radial integrals will be,

$$
\begin{aligned}
& \frac{2}{\pi} \int_{0}^{\infty} f_{p, \ell}(K) j_{n}\left(K R_{p}\right) K^{2} d K= \\
& \int_{0}^{\infty} \rho_{p, \ell}(x) x^{2}\left(\frac{2}{\pi} \int_{0}^{\infty} j_{\ell}(K x) j_{n}\left(K R_{p}\right) K^{2} d K\right) d x \\
& n=\ell+2, \ell, \ell-2
\end{aligned}
$$

Two of the three integrals in (111) are outside the bounds of analytical continuation for use of the Weber-Schafheitlin discontinuous integral. With a recursion relation for spherical Bessel functions,

$$
j_{n-1}(z)+j_{n+1}(z)=\frac{2 n+1}{z} j_{n}(z)
$$

the integrals with $j_{\ell+2}$ and $j_{\ell-2}$ in $(111)$ can be reexpressed as,

$$
\begin{aligned}
\frac{2}{\pi} \int_{0}^{\infty} j_{\ell}(K x) j_{\ell+2}\left(K R_{p}\right) K^{2} d K & = \\
\left(\frac{2 \ell+3}{R_{p}}\right) \frac{2}{\pi} \int_{0}^{\infty} j_{\ell}(K x) j_{\ell+1}\left(K R_{p}\right) K d K & -\frac{2}{\pi} \int_{0}^{\infty} j_{\ell}(K x) j_{\ell}\left(K R_{p}\right) K^{2} d K \\
\left(\frac{2 \ell-1}{R_{p}}\right) \frac{2}{\pi} \int_{0}^{\infty} j_{\ell}(K x) j_{\ell-1}\left(K R_{p}\right) K d K & -\frac{2}{\pi} \int_{0}^{\infty} j_{\ell-2}(K x) j_{\ell}\left(K R_{p}\right) K^{2} d K
\end{aligned}
$$

The integrals that have spherical Bessel functions of the same order in the integrand are Hankel's repeated integral (Watson [50] eqn (1) on p. 456) which illustrates the inverse Fourier-Bessel transform for $f_{p, \ell}(K)$.

$$
\int_{0}^{\infty} \rho_{p, \ell}(x) x^{2}\left(\frac{2}{\pi} \int_{0}^{\infty} j_{\ell}(K x) j_{\ell}\left(K R_{p}\right) K^{2} d K\right) d x=\rho_{p, \ell}\left(R_{p}\right)
$$

The other two integrals satisfy the conditions of Weber-Schafheitlin with the results the same as given by (103) and (104), but with an extra factor of $R_{p}^{-1}$ from the recursion relation (112). The radial functions from (111), but with the

Ed. 2.1 July 28, 2000 
factors $2 \ell+3$ and $2 \ell-1$ omitted since they just cancel the same terms in the denominators of factors for the products of $y_{2 M \pm} y_{\ell m \pm}$ are:

$$
\begin{aligned}
& \mathcal{R}_{p, \ell+2}\left(R_{p}\right)=\frac{1}{R_{p}^{\ell+3}} \int_{0}^{R_{p}} \rho_{p, \ell}(x) x^{\ell+2} d x \\
& \mathcal{R}_{p, \ell-2}\left(R_{p}\right)=R_{p}^{\ell-2} \int_{R_{p}}^{\infty} \rho_{p, \ell}(x) x^{1-\ell} d x
\end{aligned}
$$

The other radial function is simply $\rho_{p, \ell}\left(R_{p}\right)$ itself. Everything is now in place to give the explicit contributions of a pseudoatom at site $p$ to the electric field gradient at $\mathbf{R}_{p}$.

The field gradient expectations are related to the second order irregular spherical harmonics according to the equalities given below:

$$
\begin{array}{ll}
\left\langle\frac{3 x^{2}-r^{2}}{r^{5}}\right\rangle=\left\langle\frac{\frac{1}{2} y_{22+}-y_{20}}{r^{3}}\right\rangle & \left\langle\frac{3 x y}{r^{5}}\right\rangle=\left\langle\frac{\frac{1}{2} y_{22-}}{r^{3}}\right\rangle \\
\left\langle\frac{3 y^{2}-r^{2}}{r^{5}}\right\rangle=\left\langle\frac{-\frac{1}{2} y_{22+}-y_{20}}{r^{3}}\right\rangle & \left\langle\frac{3 x z}{r^{5}}\right\rangle=\left\langle\frac{y_{21+}}{r^{3}}\right\rangle \\
\left\langle\frac{3 z^{2}-r^{2}}{r^{5}}\right\rangle=\left\langle\frac{2 y_{20}}{r^{3}}\right\rangle & \left\langle\frac{3 y z}{r^{5}}\right\rangle=\left\langle\frac{y_{21-}}{r^{3}}\right\rangle
\end{array}
$$

Notice that this second rank tensor is traceless. The diagonal elements are shown on the lefthand side of (118). With the definitions given in (118), the radial functions (116) and (117), and the product relations for $y_{2 M \pm} y_{\ell m \pm}$, the field gradient components at $\mathbf{R}_{p}$ from pseudoatom $p$ are:

$$
\begin{aligned}
& \left\langle\frac{3 x^{2}-r^{2}}{r^{5}}\right\rangle= \\
& \sum_{\ell m \pm} \frac{3 C_{p \ell m \pm}}{4(2 \ell+1)}\left[\mathcal { R } _ { p , \ell + 2 } ( R _ { p } ) \left(y_{\ell+2 m+2 \pm}\left(\Omega_{\mathbf{R}_{p}}\right)\right.\right. \\
& \left.\quad-2 \frac{(\ell-m+2) !}{(\ell-m) !} y_{\ell+2 m \pm}\left(\Omega_{\mathbf{R}_{p}}\right)+\frac{(\ell-m+4) !}{(\ell-m) !} y_{\ell+2 m-2 \pm}\left(\Omega_{\mathbf{R}_{p}}\right)\right) \\
& \quad+\mathcal{R}_{p, \ell-2}\left(R_{p}\right)\left(y_{\ell-2 m+2 \pm}\left(\Omega_{\mathbf{R}_{p}}\right)-2 \frac{(\ell+m) !}{(\ell+m-2) !} y_{\ell-2 m \pm}\left(\Omega_{\mathbf{R}_{p}}\right)\right. \\
& \left.\quad+\frac{(\ell+m) !}{(\ell+m-4) !} y_{\ell-2 m-2 \pm}\left(\Omega_{\mathbf{R}_{p}}\right)\right) \\
& \left.\quad-\frac{4(2 \ell+1)}{3}\left(\frac{3 X_{p}^{2}-R_{p}^{2}}{R_{p}^{5}}\right) \rho_{p, \ell}\left(R_{p}\right) y_{\ell m \pm}\left(\Omega_{\mathbf{R}_{p}}\right)\right]
\end{aligned}
$$

Ed. 2.1 July 28, 2000 


$$
\begin{aligned}
\left\langle\frac{3 y^{2}-r^{2}}{r^{5}}\right\rangle= & \sum_{\ell m \pm} \frac{3 C_{p \ell m \pm}}{4(2 \ell+1)}\left[-\mathcal{R}_{p, \ell+2}\left(R_{p}\right)\left(y_{\ell+2 m+2 \pm}\left(\Omega_{\mathbf{R}_{p}}\right)\right.\right. \\
+ & \left.2 \frac{(\ell-m+2) !}{(\ell-m) !} y_{\ell+2 m \pm}\left(\Omega_{\mathbf{R}_{p}}\right)+\frac{(\ell-m+4) !}{(\ell-m) !} y_{\ell+2 m-2 \pm}\left(\Omega_{\mathbf{R}_{p}}\right)\right) \\
- & \mathcal{R}_{p, \ell-2}\left(R_{p}\right)\left(y_{\ell-2 m+2 \pm}\left(\Omega_{\mathbf{R}_{p}}\right)+2 \frac{(\ell+m) !}{(\ell+m-2) !} y_{\ell-2 m \pm}\left(\Omega_{\mathbf{R}_{p}}\right)\right. \\
+ & \left.\frac{(\ell+m) !}{(\ell+m-4) !} y_{\ell-2 m-2 \pm}\left(\Omega_{\mathbf{R}_{p}}\right)\right) \\
& \left.-\frac{4(2 \ell+1)}{3}\left(\frac{3 Y_{p}^{2}-R_{p}^{2}}{R_{p}^{5}}\right) \rho_{p, \ell}\left(R_{p}\right) y_{\ell m \pm}\left(\Omega_{\mathbf{R}_{p}}\right)\right]
\end{aligned}
$$

$$
\begin{aligned}
\left\langle\frac{3 z^{2}-r^{2}}{r^{5}}\right\rangle=\sum_{\ell m \pm} & \frac{3 C_{p \ell m \pm}}{2 \ell+1}\left[\mathcal{R}_{p, \ell+2}\left(R_{p}\right) \frac{(\ell-m+2) !}{(\ell-m) !} y_{\ell+2 m \pm}\left(\Omega_{\mathbf{R}_{p}}\right)\right. \\
+ & \mathcal{R}_{p, \ell-2}\left(R_{p}\right) \frac{(\ell+m) !}{(\ell+m-2) !} y_{\ell-2 m \pm}\left(\Omega_{\mathbf{R}_{p}}\right) \\
& \left.-\frac{(2 \ell+1)}{3}\left(\frac{3 Z_{p}^{2}-R_{p}^{2}}{R_{p}^{5}}\right) \rho_{p, \ell}\left(R_{p}\right) y_{\ell m \pm}\left(\Omega_{\mathbf{R}_{p}}\right)\right]
\end{aligned}
$$

$$
\begin{aligned}
\left\langle\frac{3 x y}{r^{5}}\right\rangle=\sum_{\ell m \pm} \frac{ \pm 3 C_{p \ell m \pm}}{4(2 \ell+1)}\left[\mathcal { R } _ { p , \ell + 2 } ( R _ { p } ) \left(y_{\ell+2 m+2 \mp}\left(\Omega_{\mathbf{R}_{p}}\right)\right.\right. \\
\left.\quad-\frac{(\ell-m+4) !}{(\ell-m) !} y_{\ell+2 m-2 \mp}\left(\Omega_{\mathbf{R}_{p}}\right)\right) \\
+\mathcal{R}_{p, \ell-2}\left(R_{p}\right)\left(y_{\ell-2 m+2 \mp}\left(\Omega_{\mathbf{R}_{p}}\right)\right. \\
\left.\quad-\frac{(\ell+m) !}{(\ell+m-4) !} y_{\ell-2 m-2 \mp}\left(\Omega_{\mathbf{R}_{p}}\right)\right) \\
\left.\quad-4(2 \ell+1)\left(\frac{X_{p} Z_{p}}{R_{p}^{5}}\right) \rho_{p, \ell}\left(R_{p}\right) y_{\ell m \pm}\left(\Omega_{\mathbf{R}_{p}}\right)\right]
\end{aligned}
$$

Ed. 2.1 July 28, 2000 


$$
\begin{aligned}
& \left\langle\frac{3 x z}{r^{5}}\right\rangle=\sum_{\ell m \pm} \frac{3 C_{p \ell m \pm}}{2(2 \ell+1)}\left[\mathcal { R } _ { p , \ell + 2 } ( R _ { p } ) ( \ell - m + 1 ) \left(y_{\ell+2 m+1 \pm}\left(\Omega_{\mathbf{R}_{p}}\right)\right.\right. \\
& \left.-\frac{(\ell-m+3) !}{(\ell-m+1) !} y_{\ell+2 m-1 \pm}\left(\Omega_{\mathbf{R}_{p}}\right)\right) \\
& -\mathcal{R}_{p, \ell-2}\left(R_{p}\right)(\ell+m)\left(y_{\ell-2 m+1 \pm}\left(\Omega_{\mathbf{R}_{p}}\right)\right. \\
& \left.-\frac{(\ell+m-1) !}{(\ell+m-3) !} y_{\ell-2 m-1 \pm}\left(\Omega_{\mathbf{R}_{p}}\right)\right) \\
& \left.-2(2 \ell+1)\left(\frac{X_{p} Z_{p}}{R_{p}^{5}}\right) \rho_{p, \ell}\left(R_{p}\right) y_{\ell m \pm}\left(\Omega_{\mathbf{R}_{p}}\right)\right] \\
& \left\langle\frac{3 y z}{r^{5}}\right\rangle=\sum_{\ell m \pm} \frac{ \pm 3 C_{p \ell m \pm}}{2(2 \ell+1)}\left[\mathcal { R } _ { p , \ell + 2 } ( R _ { p } ) ( \ell - m + 1 ) \left(y_{\ell+2 m+1 \mp}\left(\Omega_{\mathbf{R}_{p}}\right)\right.\right. \\
& \left.+\frac{(\ell-m+3) !}{(\ell-m+1) !} y_{\ell+2 m-2 \mp}\left(\Omega_{\mathbf{R}_{p}}\right)\right) \\
& -\mathcal{R}_{p, \ell-2}\left(R_{p}\right)(\ell+m)\left(y_{\ell-2 m+1 \mp}\left(\Omega_{\mathbf{R}_{p}}\right)\right. \\
& \left.+\frac{(\ell+m-1) !}{(\ell+m-3) !} y_{\ell-2 m-1 \mp}\left(\Omega_{\mathbf{R}_{p}}\right)\right) \\
& \left.-2(2 \ell+1)\left(\frac{Y_{p} Z_{p}}{R_{p}^{5}}\right) \rho_{p, \ell}\left(R_{p}\right) y_{\ell m \pm}\left(\Omega_{\mathbf{R}_{p}}\right)\right]
\end{aligned}
$$

The expressions given in (119) through (124) are coded in VALRAY for a pseudoatom calculation of the electric field gradient at $\mathbf{R}_{p}$, which need not be a nuclear site.

\section{C.4 Gradient of the Electron Density}

Spherical surface harmonics are useful functions for mathematical analyses such as Fourier transformations. For computational work, however, the Cartesian representations, such as those listed in Tables 1 to 3 in section 2.3 of this manual, are preferable. The angular functions in direction cosines are proportional to the spherical surface harmonics. A pseudoatom basis function can be represented by

$$
\rho_{\ell m \pm}(\mathbf{r})=\rho_{\ell}(r) \mathrm{A}_{\ell m \pm}\left(\mathrm{q}_{\mathrm{x}}, \mathrm{q}_{\mathrm{y}}, \mathrm{q}_{\mathrm{z}}\right) / 4 \pi
$$

The function $\mathrm{A}_{\ell m \pm}$ is computed in both the least squares and properties packages (VALLSQ and FOPROP, respectively) of VALRAY. For the gradient of the pseudoatom basis function, the $\alpha$ component (with $\alpha=x, y, z$ ) is

$$
\frac{\partial \rho_{\ell m \pm}(\mathbf{r})}{\partial \alpha}=\left[\mathrm{q}_{\alpha} \rho_{\ell}^{\prime}(r) \mathrm{A}_{\ell m \pm}+\rho_{\ell}(r) \frac{\partial \mathrm{A}_{\ell m \pm}}{\partial \alpha}\right] / 4 \pi
$$

Ed. 2.1 July 28,2000 
where in (126)

$$
\rho_{\ell}^{\prime}(r)=\frac{d \rho_{\ell}(r)}{d r}
$$

and

$$
\frac{\partial \mathrm{A}_{\ell m \pm}}{\partial \alpha}=\frac{\partial \mathrm{A}_{\ell m \pm}}{\partial \mathrm{q}_{\mathrm{x}}} \frac{\partial \mathrm{q}_{\mathrm{x}}}{\partial \alpha}+\frac{\partial \mathrm{A}_{\ell m \pm}}{\partial \mathrm{q}_{\mathrm{y}}} \frac{\partial \mathrm{q}_{\mathrm{y}}}{\partial \alpha}+\frac{\partial \mathrm{A}_{\ell m \pm}}{\partial \mathrm{q}_{\mathrm{z}}} \frac{\partial \mathrm{q}_{\mathrm{z}}}{\partial \alpha}
$$

A function routine in FOPROP computes the vector components $\partial \mathrm{A}_{\ell m \pm} / \partial \mathrm{q}_{\alpha}$. The partial derivatives of the direction cosines $\mathrm{q}_{\beta}$ with respect to a coordinate $\alpha$ in (128) is related to other direction cosines and the inverse of $r$ via the relation,

$$
\frac{\partial \mathrm{q}_{\beta}}{\partial \alpha}=\frac{\delta_{\alpha \beta}-\mathrm{q}_{\alpha} \mathrm{q}_{\beta}}{r}
$$

FOPROP also includes routines to compute $\rho_{\ell}^{\prime}(r)$ in (127) for some of the functions given in Appendix A.

\section{C.5 Hessian of the Electron Density}

For computation of the Hessian of the pseudoatom bases, the Cartesian representation for $\rho_{\ell m \pm}(\mathbf{r})$ is exploited much the same way as done for the gradient. In terms of $\rho_{\ell}(r), \mathrm{A}_{\ell m \pm}(\mathbf{q})$ and assorted partial derivatives, the Hessian of a pseudoatom basis is,

$$
\begin{aligned}
& \frac{\partial^{2} \rho_{\ell m \pm}(\mathbf{r})}{\partial \alpha \partial \beta}=\left[\mathrm{q}_{\alpha} \mathrm{q}_{\beta} \rho_{\ell}^{\prime \prime}(r) \mathrm{A}_{\ell m \pm}\right. \\
& +\rho_{\ell}^{\prime}(r)\left(\frac{\delta_{\alpha \beta}-\mathrm{q}_{\alpha} \mathrm{q}_{\beta}}{r} \mathrm{~A}_{\ell m \pm}+\mathrm{q}_{\alpha} \frac{\partial \mathrm{A}_{\ell m \pm}}{\partial \beta}+\mathrm{q}_{\beta} \frac{\partial \mathrm{A}_{\ell m \pm}}{\partial \alpha}\right) \\
& \left.+\rho_{\ell}(r) \frac{\partial^{2} \mathrm{~A}_{\ell m \pm}}{\partial \alpha \partial \beta}\right] / 4 \pi \text {. }
\end{aligned}
$$

An explicit form of the partial derivatives of the A functions with respect to a coordinate, $\alpha$, is given in (128). The second order partial derivative term that involves the A function contains factors of the type

$$
\frac{\partial^{2} \mathrm{~A}_{\ell m \pm}}{\partial \mathrm{q}_{\alpha} \partial \mathrm{q}_{\beta}}
$$

These tensor elements are calculated by a function routine in FOPROP. The routines that compute $\mathrm{A}_{\ell m \pm}, \partial \mathrm{A}_{\ell m \pm} / \partial \mathrm{q}_{\alpha}$, and $\partial^{2} \mathrm{~A}_{\ell m \pm} / \partial \mathrm{q}_{\alpha} \partial \mathrm{q}_{\beta}$ in VALRAY are restricted to a maximum $\ell$ of seven.

Ed. 2.1 July 28, 2000 


\section{C.6 Laplacian of the Electron Density}

The Laplacian operator in spherical polar coordinates is,

$$
\nabla^{2}=\frac{1}{r^{2}}\left[\frac{\partial}{\partial r}\left(r^{2} \frac{\partial}{\partial r}\right)+\frac{\partial}{\partial \eta}\left(\left(1-\eta^{2}\right) \frac{\partial}{\partial \eta}\right)+\frac{1}{\left(1-\eta^{2}\right)} \frac{\partial^{2}}{\partial \phi^{2}}\right]
$$

A gsf basis function in VALRAY is $i^{\ell} f_{\ell}(K) y_{\ell m \pm}\left(\Omega_{\mathbf{K}}\right)$ with a corresponding direct space function $\rho_{\ell}(r) y_{\ell m \pm}\left(\Omega_{\mathbf{r}}\right) / 4 \pi$. In the Laplacian operator shown above, only the $r$ derivative terms work on $\rho_{\ell}(r)$, whereas the $\eta$ and $\phi$ derivatives will apply only to $y_{\ell m \pm}$. The radial part in $r$ from (131) gives,

$$
\frac{\partial}{\partial r}\left(r^{2} \frac{\partial \rho_{\ell}(r)}{\partial r}\right)=r^{2} \rho_{\ell}^{\prime \prime}(r)+2 r \rho_{\ell}^{\prime}(r)
$$

With,

$$
y_{\ell m \pm}(\Omega)=\mathrm{P}_{\ell}^{m}(\eta)\left\{\begin{array}{l}
\cos m \phi \\
\sin m \phi
\end{array}\right.
$$

the $\eta$ dependent part from (131) is,

$$
\left(1-\eta^{2}\right) \frac{\partial^{2} \mathrm{P}_{\ell}^{m}(\eta)}{\partial \eta^{2}}-2 \eta \frac{\partial \mathrm{P}_{\ell}^{m}(\eta)}{\partial \eta}=\left[\frac{m^{2}}{\left(1-\eta^{2}\right)}-\ell(\ell+1)\right] \mathrm{P}_{\ell}^{m}(\eta) .
$$

The right hand side of (134) completes the defining differential equation for an associated Legendre function. For the $\phi$ dependence,

$$
\frac{1}{\left(1-\eta^{2}\right)} \frac{\partial^{2}}{\partial \phi^{2}}\left\{\begin{array}{c}
\cos m \phi \\
\sin m \phi
\end{array}=-\frac{m^{2}}{\left(1-\eta^{2}\right)}\left\{\begin{array}{c}
\cos m \phi \\
\sin m \phi
\end{array}\right.\right.
$$

By summing (132), (134) and (135), and dividing by $r^{2}$ the Laplacian of the pseudoatom basis functions is,

$$
\nabla^{2} \rho_{\ell m \pm}(\mathbf{r})=\left[\rho_{\ell}^{\prime \prime}(r)+2 \frac{\rho_{\ell}^{\prime}(r)}{r}-\ell(\ell+1) \frac{\rho_{\ell}(r)}{r^{2}}\right] y_{\ell m \pm}\left(\Omega_{\mathbf{r}}\right) / 4 \pi
$$

The $r$ dependent function in (136) has been coded in VALRAY for several of the radial functions discussed in Appendix A. 